\title{
ZQP mit aktualisierter Datenbank
}

\section{Aktuelle Leitlinien und Standards auf einen Blick}

» Das Zentrum für Qualität in der Pflege (ZQP) hat seine frei zugängliche Datenbank für pflegerelevante Leitlinien und Standards aktualisiert - in Kooperation mit dem Institut für Gesundheits- und Pflegewissenschaft an der Martin-Luther-Universität HalleWittenberg. Die 161 deutsch- und englischsprachigen Dokumente sind systematisch erschlossen und mit Zusatzinformationen wie Gütehinweisen hinterlegt. Neu in die Datenbank eingegangen sind Dokumente zu den Themen Schmerzmanagement, Harnausscheidung, Mobilisierung und Lagerung sowie zur Palliativversorgung. Auch zum Thema Dekubitusprophylaxe und -behandlung bzw. Versorgung chronischer Wunden wurden weitere Arbeiten aufgenommen.

Die Untersuchung der Leitlinien und Standards aus dem deutschsprachigen Raum zeigt, dass aktuelle Dokumente wichtige methodische Qua- litätskriterien erfüllen. Überall wurde der Erarbeitungsprozess beschrieben und die Empfehlungen mehrheitlich in einem klar definierten Abstimmungsverfahren verabschiedet. „Es ist sehr positiv, dass sich der Entwicklungsprozess für Leitlinien und Standards weiter verbessert hat. Allerdings gibt es auch noch einiges zu tun. Vor allem sollte die Bewohner- und $\mathrm{Pa}$ tientenperspektive im Erarbeitungsprozess stärker berücksichtigt werden“, erklärt Dr. Ralf Suhr, Vorstandsvorsitzender des ZQP. Besonders wichtig sei es daher, geeignete Verfahren zur Einbindung von Patientenbzw. Bewohnervertretern zu entwickeln. Aus Suhrs Sicht besteht ein anderer wichtiger Ansatzpunkt für die Weiterentwicklung beim Praxistransfer: „Die Hinweise zur Umsetzung von Leitlinien und Standards bleiben eine große Herausforderung. Nur in wenigen Dokumenten werden Vorschläge zur Implementierung gemacht und entsprechende Kriterien dazu genannt."

Mit der jetzt vorgelegten Übersicht will das ZQP darum auch die Entwickler von Leitlinien und Standards unterstützen, vorhandenes Wissen aufzufinden und Impulse aus anderen Entwicklungsprozessen zu nutzen. Die Datenbank ermöglicht dafür eine einfache Recherche nach Titel, Thema, Herkunftsland oder Erscheinungsjahr der Leitlinien und Standards. Eingeschlossen sind dabei nun auch Dokumente, die zwar ohne pflegerische Beteiligung erstellt wurden, die aber Themen betreffen, in denen Pflegende ohne ärztliche Anordnung Entscheidungen treffen können oder die Maßnahmen beinhalten, die in den direkten Verantwortungsbereich der Pflegenden fallen.

\section{www.zqp.de/pflegeleitlinien}

\section{Pflegekammer: Pflichtmitgliedschaft rechtmäßig}

\section{Verwaltungsgericht Mainz lehnt Klage ab}

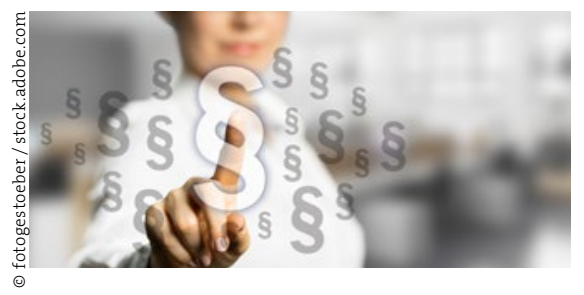

॥ Die Pflichtmitgliedschaft in einer Pflegekammer entspricht geltendem Recht. Mit dieser Einschätzung hat das Verwaltungsgericht Mainz einer Pflegerin widersprochen, die gegen die Pflichtmitgliedschaft geklagt hatte. Dem Gericht zufolge ist die Pflichtmitgliedschaft verhältnismäßig, da sie keine elementare Beschränkung der persönlichen Freiheit des einzelnen Mitgliedes darstellt.

„Das ist ein wichtiges und klärendes Signal in der Errichtungsphase der Pflegekammern in Schleswig-Holstein und Niedersachsen. Gleiches gilt für die ge- rade beginnende Diskussion um eine Pflegekammer in Nordrhein-Westfalen“, sagte Martin Dichter, Vorsitzender des DBfK Nordwest. Er äußerte die Hoffnung, das im April getroffene Urteil des Verwaltungsgerichtes Mainz könne zu einer Versachlichung der Diskussion um eine Pflegekammer in NordrheinWestfalen beitragen. Eine drängende Frage ist seiner Einschätzung nach, wie die Pflegenden in Nordrhein-Westfalen hinreichend informiert werden können. Nur so könne sichergestellt werden, dass sich die Berufsgruppe an der Diskussion beteiligt. Dagegen wünscht sich DBfK-Mann Dichter ein Ende der „Verunsicherung und Fehlinformation der Pflegenden durch Kammergegner hinsichtlich der dauerhaften Darstellung der Pflegekammer als leistungsunfähiges Bürokratiemonster“.

www.dbfk.de

\section{Brandenburg plant Umfrage zur Pflegekammer}

\section{Ministerium will Meinungsbild}

» Das brandenburgische Sozialministerium plant eine Informationskampagne zur Pflegekammer. Diese soll das Für und Wider einer Berufskammer für Pflegekräfte beleuchten und noch in der zweiten Jahreshälfte starten. Anschließend sei eine Befragung der Pflegekräfte geplant, teilte das Ministerium der Deutschen Presse-Agentur mit.

Eine Pflegekammer fordern CDU und Grüne in Brandenburg bereits seit langem. Noch im April hatten sich die Oppositionsfraktionen im Landtag für eine solche Interessenvertretung stark gemacht.

www.masgf.de 